\title{
Evaluation of Waste Transportation Routes at Gayamsari and East Semarang Subdistrict
}

\author{
Irawan Wisnu Wardhana, ${ }^{1, *}$, Wiharyanto Oktiawan ${ }^{1}$, Ganjar Samudro ${ }^{1}$, Budi Prasetyo Samadikun $^{1}$, Dickie Perdana \\ Mukti $^{1}$, and Ferba Ramadhan Khanza ${ }^{1}$
}

${ }^{1}$ Department of Environmental Engineering, Faculty of Engineering, Diponegoro University, Semarang - Indonesia

\begin{abstract}
Gayamsari and East Semarang Subdistrict are two subdistricts in Semarang City which are located side by side. According to the policy of Semarang City development in 2011-2031, Gayamsari Subdistrict has a function as an environmental center development, while East Semarang Subdistrict has the main function as office, trade and service. Preliminary studies show that in these two subdistricts the resulting of waste generation in several locations of Temporary Disposal Sites can not all be transported to Jatibarang Final Disposal Site. The purpose of this research is to examine and evaluate the existing condition of waste transportation routes in Gayamsari and East Semarang Subdistrict. This research uses survey research method, using interview, observation, documentation, and routing. Analytical techniques used are quantitative analysis and qualitative analysis. The result of this research shows that in Gayamsari and East Semarang Subdistricts, the level of service of waste transportation has not been optimum since in some locations of the Temporary Disposal Site is still found waste container which is not enough to accommodate the waste and the route far enough to the final disposal place.
\end{abstract}

Keywords: waste transportation route; Gayamsari; East Semarang.

\section{Introduction}

The increasing number of population and the increasing of society economic activity in Semarang City have an impact on the increasing amount of waste generation, as happened in Gayamsari and East Semarang Subdistrict. In order to prevent environmental problems, a good waste transport system is needed. The increasing quantity of waste generation, then the transportation system will become more complicated [1]. Important factors in the waste transport system, including quantity, type of equipment, distance, and labor needs [2]. In addition to this, waste transportation time is also an important thing in the waste transportation system [3].

Data obtained from DLH Semarang City [4], in the year 2015 waste produced by residents Gayamsari Subdistrict amounted to $286,85 \mathrm{~m}^{3}$ every day. While the waste transport capacity in the District Gayamsari is only $129,08 \mathrm{~m}^{3}$ per day. This means that the percentage of waste transportation service in Gayamsari sub district only reaches about $45 \%$. In East Semarang Subdistrict, waste production is $237,455 \mathrm{~m}^{3}$ per day, while its transportation capacity is only $189,361 \mathrm{~m}^{3}$ per day. So that the percentage rate of only $79.7 \%$ transportation services. Whereas the target of waste management system in the field of transportation according to the National Medium Term Development Plan 2014-2019 is $100 \%$, so the Government of Semarang City, especially in Gayamsari and East Semarang Subdistrict need to make efforts to improve waste service, especially in the field of waste transportation.

Considering the phenomena occurring in Gayamsari and East Semarang Subdistricts, an evaluation of the waste transportation route in the two sub-districts is needed, to increase the percentage of services in the field of current waste transportation to obtain the most optimum route [5].

The purpose of this paper is to examine and evaluate the existing condition of waste transportation routes in Gayamsari and East Semarang Subdistrict, so that it can be found a better waste transportation route (effective and efficient) in order to create low carbon society in development process.

\section{Research method}

This research uses survey research method, using interview, observation, documentation, and routing. Research site located in Gayamsari and East Semarang Subdistrict.

All garbage trucks in these two subdistricts are used as samples, 5 trucks in Gayamsari Subdistrict and 6 trucks in East Semarang Subdistrict.

Samples in Gayamsari Subdistrict were taken from 11 temporary disposal sites, that located in 7 urban villages, ie.: Pandean Lamper, Gayamsari, Siwalan, Sambirejo, Sawahbesar, Kaligawe, and Tambakrejo.

Samples in Semarang Timur Subdistrict were taken from 12 temporary disposal sites, that located in 10 urban villages, ie.: Kemijen, Rejomulyo, Mlatibaru,

* Corresponding author: irawanwisnuwardana18@gmail.com 
Mlatiharjo, Kebonagung, Bugangan, Sarirejo, Rejosari, Karangturi, Karangtempel.

\section{Result and discussion}

\subsection{Existing condition of waste transportation service in Gayamsari Subdistrict and East Semarang Subdistrict}

Based on data from the Central Bureau of Statistics [6], Gayamsari Subdistrict which has wide area $5,25 \mathrm{~km}^{2}$ consists of 7 villages, $62 \mathrm{RW}, 444 \mathrm{RT}$, and the amount of population at the end of 2016 is 74.122 people, resulting in a total amount of waste about $350,23 \mathrm{~m}^{3}$ per day, it's only can be transported as big as $276,80 \mathrm{~m}^{3}$ per day, or it is only served around $79 \%$, with the percentage composition of services as follows:

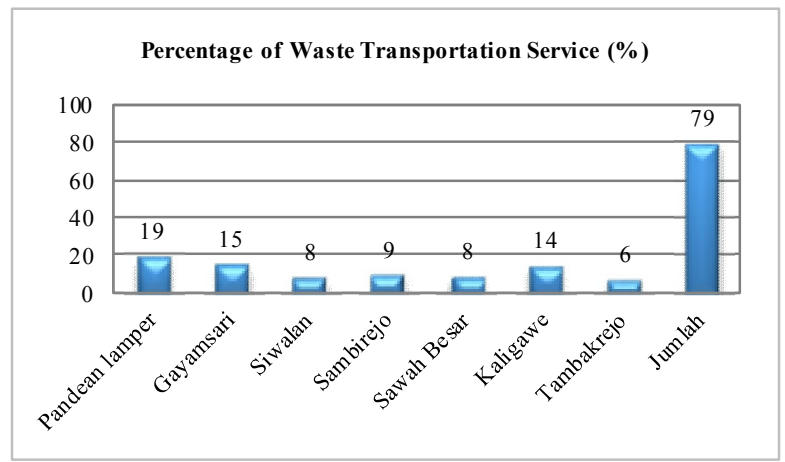

Fig. 1. Percentage of Waste Transportation Service in Gayamsari Subdistrict

Semarang Timur Subdistrict, with total area of 7,70 $\mathrm{km}^{2}$, consists of 10 villages, $77 \mathrm{RW}, 574 \mathrm{RT}$, and the amount of population at the end of 2016 is 76.574 people [7], resulting in a total amount of waste about 237,455 $\mathrm{m}^{3}$ per day, it's only can be transported as big as 189,361 $\mathrm{m}^{3}$ per day, or it is only served around $79,7 \%$, with the percentage composition of services as follows:

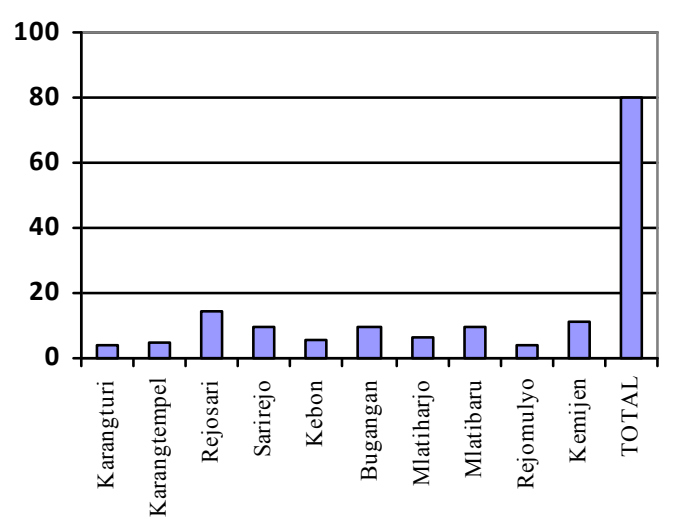

Fig. 2. Percentage of Waste Transportation Service in East Semarang Subdistrict

\subsection{Evaluation of Waste transportation route}

Enri Damanhuri states that one of the procedures for determining an effective and efficient transport system in a waste transport operation should be to use the shortest possible transport route and with the smallest possible obstacles [8].

\subsubsection{Waste Transportation Route at Gayamsari Subdistrict}

The existing trucking route from the Environment Agency of Semarang City in Gayamsari Subdistrict can be seen in the following table:

Table 1. Existing Waste Transportation Route From Gayamsari Subdistrict to TPA Jatibarang

\begin{tabular}{|c|c|c|}
\hline $\begin{array}{c}\text { Truck } \\
\text { Number }\end{array}$ & $\begin{array}{c}\text { Temporary } \\
\text { Disposal Site }\end{array}$ & Route \\
\hline \multirow{2}{*}{1} & PLTG & $\begin{array}{l}\text { Jl. Majapahit-Jl.Lamper Tengah-Jl.Tentara } \\
\text { Pelajar-Jl.Sriwijaya-Jl.Veteran- } \\
\text { Jl.Kaligarang-Jl.Simongan-Jl.Untung } \\
\text { Suropati- TPA Jatibarang }\end{array}$ \\
\hline & Kimar & $\begin{array}{l}\text { Jl.Brigjend Katamso-Jl.Kompol Maksum- } \\
\text { Jl.Sriwijaya-Jl.Veteran-Jl.Kaligarang- } \\
\text { Jl.Simongan-Jl.Untung Suropati-TPA } \\
\text { Jatibarang }\end{array}$ \\
\hline \multirow[b]{2}{*}{2} & Karangingas & $\begin{array}{l}\text { Jl.Soekarno Hatta-Jl.Dr Cipto-Jl.Brigjend } \\
\text { Katamso-Jl.Kompol Maksum-Jl.Sriwijaya- } \\
\text { Jl.Veteran-Jl.Kaligarang-Jl.Simongan- } \\
\text { Jl.Untung Suropati-TPA Jatibarang }\end{array}$ \\
\hline & Kaligawe & $\begin{array}{l}\text { J.Kaligawe Raya-Jl.Raden Patah-Jl.Dr } \\
\text { Cipto-Jl.Brigjend Katamso-Jl.Kompol } \\
\text { Maksum-Jl.Sriwijaya-Jl.Veteran- } \\
\text { Jl.Kaligarang-Jl.Simongan-Jl.Untung } \\
\text { Suropati-TPA Jatibarang }\end{array}$ \\
\hline \multirow{3}{*}{3} & Sawah Besar & $\begin{array}{l}\text { Jl.Soekarno Hatta-Jl.Dr Cipto-Jl.Brigjend } \\
\text { Katamso-Jl.Kompol Maksum-Jl.Sriwijaya- } \\
\text { Jl.Veteran-Jl.Kaligarang-Jl.Simongan- } \\
\text { Jl.Untung Suropati-TPA Jatibarang }\end{array}$ \\
\hline & Siwalan & \multirow{2}{*}{$\begin{array}{l}\text { Jl.Gajah Raya-Jl.Lamper Tengah-Jl.Tentara } \\
\text { Pelajar-Jl.Sriwijaya-Jl.Veteran- } \\
\text { Jl.Kaligarang-Jl.Simongan-Jl.Untung } \\
\text { Suropati-TPA Jatibarang }\end{array}$} \\
\hline & MAJT & \\
\hline 4 & Gempolsari & $\begin{array}{l}\text { Jl.Lamper Tengah-Jl.Tentara Pelajar- } \\
\text { Jl.Sriwijaya-Jl.Veteran-Jl.Kaligarang- } \\
\text { Jl.Simongan-Jl.Untung Suropati-TPA } \\
\text { Jatibarang }\end{array}$ \\
\hline \multirow{3}{*}{5} & Sambirejo & $\begin{array}{l}\text { Jl.Gajah Raya-Jl.Lamper Tengah-Jl.Tentara } \\
\text { Pelajar-Jl.Sriwijaya-Jl.Veteran- } \\
\text { Jl.Kaligarang-Jl.Simongan-Jl.Untung } \\
\text { Suropati-TPA Jatibarang }\end{array}$ \\
\hline & Tambakrejo & \multirow{2}{*}{$\begin{array}{l}\text { J.Kaligawe Raya-Jl.Raden Patah-Jl.Dr } \\
\text { Cipto-Jl.Brigjend Katamso-Jl.Kompol } \\
\text { Maksum-Jl.Sriwijaya-Jl.Veteran- } \\
\text { J1.Kaligarang-Jl.Simongan-Jl.Untung } \\
\text { Suropati-TPA Jatibarang }\end{array}$} \\
\hline & Pasar Waru & \\
\hline
\end{tabular}

The waste transport route in Table 1 is the usual garbage route passed by garbage trucks from Gayamsari Subdistrict to TPA Jatibarang. The distance and waste transportation time of the exisiting route can be seen in Table 2 below:

Table 2. Distance and Waste Transportation Time of Existing Route in Gayamsari Subdistrict

\begin{tabular}{|c|c|c|c|}
\hline Truck Number & $\begin{array}{c}\text { Temporary } \\
\text { Disposal Site }\end{array}$ & Distance (km) & Time (minute) \\
\hline \multirow{2}{*}{1} & PLTG & 27,45 & 90 \\
\cline { 2 - 4 } & Kimar & 27,3 & 85 \\
\hline \multirow{2}{*}{2} & Karangingas & 34,26 & 100 \\
\cline { 2 - 4 } & Kaligawe & 37,33 & 110 \\
\hline \multirow{3}{*}{3} & Sawah Besar & 35,81 & 105 \\
\cline { 2 - 4 } & Siwalan & 30,55 & 104 \\
\cline { 2 - 4 } & MAJT & 30,51 & 103 \\
\hline \multirow{2}{*}{4} & Gempolsari & 27,1 & 90 \\
\hline \multirow{3}{*}{5} & Sambirejo & 29,82 & 92 \\
\cline { 2 - 4 } & Tambakrejo & 35,17 & 98 \\
\cline { 2 - 4 } & Pasar Waru & 36,14 & 101 \\
\hline
\end{tabular}


The distance and time in Table 2 is based on the existing route from the Environment Agency of Semarang City. The furthest remaining transport route is on the truck number 2 . The distance of haul on the existing route can still be streamlined by changing the shorter route lanes and fewer traffic constraints. More efficient transport routes can be seen in Table 3 below :

Table 3. Waste Transportation Route After Efficiency

\begin{tabular}{|c|c|c|}
\hline $\begin{array}{c}\text { Truck } \\
\text { Number }\end{array}$ & $\begin{array}{c}\text { Temporary } \\
\text { Disposal Site }\end{array}$ & Route \\
\hline \multirow[t]{2}{*}{1} & PLTG & $\begin{array}{l}\text { Jl.Majapahit-Jl.Brigjend } \\
\text { Katamso-Jl.Admodirono- } \\
\text { Jl.Singosari Raya-Jl.Sriwijaya- } \\
\text { Jl.Veteran-Jl.Kaligarang- } \\
\text { J1.Simongan-Jl.Untung Suropati- } \\
\text { TPA Jatibarang }\end{array}$ \\
\hline & Kimar & $\begin{array}{l}\text { Jl.Brigjend Katamso-Jl.Admodirono- } \\
\text { Jl.Singosari Raya-Jl.Sriwijaya- } \\
\text { Jl.Veteran-Jl.Kaligarang-JI.Simongan- } \\
\text { Jl.Untung Suropati-TPA Jatibarang }\end{array}$ \\
\hline \multirow[t]{2}{*}{2} & Karangingas & $\begin{array}{l}\text { Jl.Soekarno Hatta-Jl.Dr Cipto- } \\
\text { Jl.Brigjend Katamso- } \\
\text { Jl.Admodirono-Jl.Singosari Raya- } \\
\text { Jl.Sriwijaya-Jl.Veteran- } \\
\text { Jl.Kaligarang-Jl.Simongan- } \\
\text { Jl.Untung Suropati-TPA } \\
\text { Jatibarang }\end{array}$ \\
\hline & Kaligawe & $\begin{array}{l}\text { Jl.Kaligawe Raya-Jl.Raden Patah-Jl.Dr } \\
\text { Cipto-Jl.Brigjend Katamso- } \\
\text { Jl.Admodirono-Jl.Singosari Raya- } \\
\text { Jl.Sriwijaya-Jl.Veteran-JI.Kaligarang- } \\
\text { Jl.Simongan-Jl.Untung Suropati-TPA } \\
\text { Jatibarang }\end{array}$ \\
\hline \multirow[t]{3}{*}{3} & Sawah Besar & $\begin{array}{l}\text { Jl.Soekarno Hatta-Jl.Dr Cipto- } \\
\text { Jl.Brigjend Katamso- } \\
\text { Jl.Admodirono-Jl.Singosari Raya- } \\
\text { Jl.Sriwijaya-Jl.Veteran- } \\
\text { Jl.Kaligarang-Jl.Simongan- } \\
\text { Jl.Untung Suropati-TPA } \\
\text { Jatibarang }\end{array}$ \\
\hline & Siwalan & \multirow{2}{*}{$\begin{array}{l}\text { Jl.Gajah Raya-Jl.Majapahit-Jl.Brigjend } \\
\text { Katamso-Jl.Admodirono-Jl.Singosari } \\
\text { Raya-JI.Sriwijaya-Jl.Veteran- } \\
\text { Jl.Kaligarang-JIISimongan-Jl.Untung } \\
\text { Suropati-TPA Jatibarang }\end{array}$} \\
\hline & MAJT & \\
\hline 4 & Gempolsari & $\begin{array}{l}\text { Jl.Majapahit-Jl.Brigjend } \\
\text { Katamso-Jl.Admodirono- } \\
\text { Jl.Singosari Raya-Jl.Sriwijaya- } \\
\text { Jl.Veteran-Jl.Kaligarang- } \\
\text { Jl.Simongan-Jl.Untung Suropati- } \\
\text { TPA Jatibarang }\end{array}$ \\
\hline \multirow[t]{3}{*}{5} & Sambirejo & $\begin{array}{l}\text { Jl.Gajah Raya-Jl.Lamper Tengah- } \\
\text { Jl.Tentara Pelar-Jl.Sriwijaya- } \\
\text { Jl.Veteran-Jl.Kaligarang- } \\
\text { Jl.Simongan-Jl.Untung Suropati } \\
\text { ke TPA Jatibarang }\end{array}$ \\
\hline & Tambakrejo & \multirow[b]{2}{*}{$\begin{array}{l}\text { J.Kaligawe Raya-Jl.Raden Patah- } \\
\text { Jl.Dr Cipto-Jl.Brigjend Katamso- } \\
\text { Jl.Admodirono-Jl.Singosari Raya- } \\
\text { Jl.Sriwijaya-Jl.Veteran- } \\
\text { Jl.Kaligarang-Jl.Simongan- } \\
\text { Jl.Untung Suropati-TPA } \\
\text { Jatibarang }\end{array}$} \\
\hline & Pasar Waru & \\
\hline
\end{tabular}

Looking at the results in Table 3, there was a change of waste transport route on the road passed by garbage truck through Admodirono Road and Singosari Raya Road to go to Sriwijaya Street where this path is a road with little roadblock (traffic jam and less traffic light). The distance and time of transporting waste through the efficient routes can be seen in Table 4 below.
Table 4. Distance and Waste Transportation Time After Efficiency

\begin{tabular}{|c|l|c|c|}
\hline $\begin{array}{c}\text { Truck } \\
\text { Number }\end{array}$ & $\begin{array}{c}\text { Temporary } \\
\text { Disposal Site }\end{array}$ & $\begin{array}{c}\text { Distance } \\
(\mathbf{k m})\end{array}$ & $\begin{array}{c}\text { Time } \\
(\mathbf{m i n u t e})\end{array}$ \\
\hline \multirow{2}{*}{1} & PLTG & 26,8 & 69 \\
\cline { 2 - 4 } & Kimar & 26,65 & 67 \\
\hline \multirow{2}{*}{2} & Karangingas & 33,16 & 81 \\
\cline { 2 - 4 } & Kaligawe & 36,69 & 90 \\
\hline \multirow{3}{*}{3} & Sawah Besar & 35,16 & 85 \\
\cline { 2 - 4 } & Siwalan & 29,63 & 78 \\
\cline { 2 - 4 } & MAJT & 29,61 & 78 \\
\hline \multirow{2}{*}{4} & Gempolsari & 26,6 & 67 \\
\hline \multirow{2}{*}{5} & Sambirejo & 27,68 & 78 \\
\cline { 2 - 4 } & Tambakrejo & 34,49 & 81 \\
\cline { 2 - 4 } & Pasar Waru & 35,46 & 85 \\
\hline
\end{tabular}

The difference between the distance and time of the truck transport can be determined by comparing the distance and time of the existing route with the distance and time of the efficiency route. The difference between the distance and time of the existing route with the efficiency route can be seen in Table 5 below :

Table 5. Distance and Time Difference After Efficiency

\begin{tabular}{|c|c|c|c|}
\hline \multirow[b]{2}{*}{$\begin{array}{c}\text { Truck } \\
\text { Number }\end{array}$} & \multirow{2}{*}{$\begin{array}{c}\text { Temporary } \\
\text { Disposal Site }\end{array}$} & \multicolumn{2}{|c|}{ Difference } \\
\hline & & $\begin{array}{c}\text { Distance } \\
(\mathrm{km})\end{array}$ & $\begin{array}{c}\text { Time } \\
\text { (minute) }\end{array}$ \\
\hline \multirow{2}{*}{1} & PLTG & 0,65 & 21 \\
\hline & Kimar & 0,65 & 18 \\
\hline \multirow{2}{*}{2} & Karangingas & 1,2 & 19 \\
\hline & Kaligawe & 0,65 & 20 \\
\hline \multirow{3}{*}{3} & Sawah Besar & 0,65 & 20 \\
\hline & Siwalan & 0,92 & 26 \\
\hline & MAJT & 0,9 & 25 \\
\hline 4 & Gempolsari & 0,5 & 23 \\
\hline \multirow{3}{*}{5} & Sambirejo & 2,14 & 14 \\
\hline & Tambakrejo & 0,68 & 17 \\
\hline & Pasar Waru & 0,68 & 16 \\
\hline
\end{tabular}

The result of distance and time difference calculation in Table 5 shows that the largest distance difference in truck with number 5 serving TPS Sambirejo to TPA Jatibarang has distance of $2.14 \mathrm{~km}$. While the largest time difference of 26 minutes is on truck number 3 that serves TPS Siwalan to TPA Jatibarang.

\subsubsection{Waste Transportation Route at East Semarang Subdistrict}

The existing trucking route from the Environment Agency of Semarang City in East Semarang Subdistrict can be seen in the following table: 
Table 6. Existing Waste Transportation Route From East Semarang Subdistrict to TPA Jatibarang

\begin{tabular}{|c|c|c|}
\hline $\begin{array}{c}\text { Truck } \\
\text { Number }\end{array}$ & $\begin{array}{c}\text { Temporary } \\
\text { Disposal Site }\end{array}$ & Route \\
\hline \multirow{3}{*}{1} & Pasar Rejomulyo & $\begin{array}{l}\text { Jl. Pengapon-Jl. Raden Patah-Jl. } \\
\text { Widoharjo-Jl. Dr. Cipto-Jl. Kompol } \\
\text { Maksum-Jl. Sriwijaya-Jl.Veteran- } \\
\text { Jl.Kaligarang, Jl.Simongan- } \\
\text { Jl.Untung Suropati-TPA Jatibarang }\end{array}$ \\
\hline & Manisharjo & $\begin{array}{l}\text { J1. Raden Patah-Jl. Widoharjo-Jl. Dr. } \\
\text { Cipto-Jl. Kompol Maksum-Jl. Sriwijaya- } \\
\text { Jl.Veteran-Jl.Kaligarang, Jl.Simongan, } \\
\text { Jl.Untung Suropati- TPA Jatibarang }\end{array}$ \\
\hline & Pasar Waru & $\begin{array}{l}\text { Jl. Kaligawe Raya-Jl. Raden Patah- Jl. } \\
\text { Widoharjo-Jl. Dr. Cipto-Jl. Kompol } \\
\text { Maksum-JI. Sriwijaya, Jl.Veteran- } \\
\text { Jl.Kaligarang, Jl.Simongan-Jl.Untung } \\
\text { Suropati- TPA Jatibarang }\end{array}$ \\
\hline \multirow[b]{2}{*}{2} & Pasar Dargo & $\begin{array}{l}\text { JI. Dargo-Jl. Dr. Cipto- Jl. Kompol } \\
\text { Maksum- Jl. Sriwijaya- Jl.Veteran- } \\
\text { Jl.Kaligarang- Jl.Simongan- Jl.Untung } \\
\text { Suropati- TPA Jatibarang }\end{array}$ \\
\hline & Pasar Karimata & $\begin{array}{l}\text { Jl. Pancakarya- Jl. RA. Kartini- Dr. } \\
\text { Cipto- Jl. Kompol Maksum- Jl. } \\
\text { Sriwijaya- Jl.Veteran- Jl.Kaligarang } \\
\text { - Jl.Simongan-Jl.Untung Suropati, } \\
\text { TPA Jatibarang }\end{array}$ \\
\hline 3 & Rumah Pompa & $\begin{array}{l}\text { Jl. Barito- Jl. RA. Kartini- Dr. Cipto- Jl. } \\
\text { Kompol Maksum- Jl. Sriwijaya- } \\
\text { Jl.Veteran- Jl.Kaligarang- Jl.Simongan- } \\
\text { Jl.Untung Suropati- TPA Jatibarang }\end{array}$ \\
\hline 4 & Mlatiharjo & $\begin{array}{l}\text { Jl. Barito-Jl. Citarum- Jl. RA. Kartini- } \\
\text { Dr. Cipto- Jl. Kompol Maksum- Jl. } \\
\text { Sriwijaya- Jl.Veteran- Jl.Kaligarang- } \\
\text { Jl.Simongan- Jl.Untung Suropati- TPA } \\
\text { Jatibarang }\end{array}$ \\
\hline \multirow[b]{2}{*}{5} & Karang Tempel & $\begin{array}{l}\text { Jl. Barito- Jl. Halmahera Raya- Dr. } \\
\text { Cipto- Jl. Kompol Maksum- Jl. } \\
\text { Sriwijaya- Jl.Veteran- Jl.Kaligarang- } \\
\text { Jl.Simongan- Jl.Untung Suropati- TPA } \\
\text { Jatibarang }\end{array}$ \\
\hline & Mlatibaru & $\begin{array}{l}\text { Jl. Indragiri- Jl. Raden Patah- Jl. } \\
\text { Widoharjo- Jl. Dr. Cipto- Jl. Kompol } \\
\text { Maksum- Jl. Sriwijaya- Jl.Veteran- } \\
\text { Jl.Kaligarang- Jl.Simongan- Jl.Untung } \\
\text { Suropati- TPA Jatibarang }\end{array}$ \\
\hline \multirow{3}{*}{6} & Tirtoyoso I & $\begin{array}{l}\text { Jl. Barito- Jl. RA. Kartini- Dr. Cipto- Jl. } \\
\text { Kompol Maksum- Jl. Sriwijaya- } \\
\text { Jl.Veteran- Jl.Kaligarang- Jl.Simongan- } \\
\text { Jl.Untung Suropati- TPA Jatibarang }\end{array}$ \\
\hline & Tirtoyoso IV & \multirow{2}{*}{$\begin{array}{l}\text { Jl. Barito-Jl. Citarum- Jl. RA. } \\
\text { Kartini- Dr. Cipto-Jl. Kompol } \\
\text { Maksum- Jl. Sriwijaya- Jl.Veteran- } \\
\text { Jl.Kaligarang- Jl.Simongan- } \\
\text { Jl.Untung Suropati- TPA Jatibarang }\end{array}$} \\
\hline & Bugangan & \\
\hline
\end{tabular}

The waste transport route in Table 6 is the usual garbage route passed by garbage trucks from East Semarang Subdistrict to TPA Jatibarang. The distance and waste transportation time of the exisiting route can be seen in Table 7 below :

Table 7. Distance and Waste Transportation Time For Existing Route in East Semarang Subdistrict

\begin{tabular}{|c|l|c|r|}
\hline $\begin{array}{c}\text { Truck } \\
\text { Number }\end{array}$ & $\begin{array}{c}\text { Temporary } \\
\text { Disposal Site }\end{array}$ & $\begin{array}{c}\text { Distance } \\
(\mathbf{k m})\end{array}$ & $\begin{array}{c}\text { Time } \\
\text { (minute) }\end{array}$ \\
\hline \multirow{3}{*}{1} & Pasar Rejomulyo & 34,4 & 79,8 \\
\cline { 2 - 4 } & Manisharjo & 32,8 & 81 \\
\cline { 2 - 4 } & Pasar Waru & 35 & 79,2 \\
\hline \multirow{2}{*}{2} & Pasar Dargo & 29,4 & 81 \\
\cline { 2 - 4 } & Pasar Karimata & 28,4 & 87,6 \\
\hline 3 & Rumah Pompa & 28 & 82,8 \\
\hline 4 & Mlatiharjo & 32,4 & 79,8 \\
\hline \multirow{2}{*}{5} & Karang Tempel & 26 & 84,6 \\
\cline { 2 - 4 } & Mlatibaru & 33,8 & 79,2 \\
\hline
\end{tabular}

\begin{tabular}{|c|l|c|c|}
\hline $\begin{array}{c}\text { Truck } \\
\text { Number }\end{array}$ & $\begin{array}{c}\text { Temporary } \\
\text { Disposal Site }\end{array}$ & $\begin{array}{c}\text { Distance } \\
\text { (km) }\end{array}$ & $\begin{array}{c}\text { Time } \\
\text { (minute) }\end{array}$ \\
\hline \multirow{3}{*}{6} & Tirtoyoso I & 29,8 & 83,4 \\
\cline { 2 - 4 } & Tirtoyoso IV & 29,6 & 79,8 \\
\cline { 2 - 4 } & Bugangan & 30 & 86,4 \\
\hline
\end{tabular}

The distance and time in Table 7 is based on the existing route from the Environment Agency of Semarang City. The furthest remaining transport route is on the truck number 1 . The distance of haul on the existing route can can still be made more efficient by changing the shorter route lanes and fewer traffic constraints. More efficient transport routes can be seen in Table 8 below :

Table 8. Waste Transportation Route in East Semarang Subdistrict After Efficiency

\begin{tabular}{|c|c|c|}
\hline $\begin{array}{c}\text { Truck } \\
\text { Number }\end{array}$ & $\begin{array}{c}\text { Temporary } \\
\text { Disposal Site }\end{array}$ & Route \\
\hline \multirow{3}{*}{1} & Pasar Rejomulyo & $\begin{array}{l}\text { Jl. Pengapon- Jl. Raden Patah- Jl. } \\
\text { Widoharjo- Jl. Dr. Cipto-Jl. } \\
\text { Brigjend Katamso- } \\
\text { Jl.Admodirono- Jl.Singosari } \\
\text { Raya- Jl. Sriwijaya- Jl.Veteran- } \\
\text { Jl.Kaligarang- Jl.Simongan- } \\
\text { Jl.Untung Suropati- TPA } \\
\text { Jatibarang }\end{array}$ \\
\hline & Manisharjo & $\begin{array}{l}\text { J1. Raden Patah- J1. Widoharjo- J1. } \\
\text { Dr. Cipto- Jl. Brigjend Katamso- } \\
\text { J1.Admodirono- Jl.Singosari } \\
\text { Raya- Jl. Sriwijaya- J1.Veteran- } \\
\text { J1.Kaligarang- J1.Simongan- } \\
\text { J1.Untung Suropati- TPA } \\
\text { Jatibarang }\end{array}$ \\
\hline & Pasar Waru & $\begin{array}{l}\text { Jl. Kaligawe Raya- Jl. Raden } \\
\text { Patah- Jl. Widoharjo- Jl. Dr. } \\
\text { Cipto- Jl. Brigjend Katamso- } \\
\text { Jl.Admodirono- Jl.Singosari } \\
\text { Raya- Jl. Sriwijaya- Jl.Veteran- } \\
\text { Jl.Kaligarang- Jl.Simongan- } \\
\text { Jl.Untung Suropati- TPA } \\
\text { Jatibarang }\end{array}$ \\
\hline \multirow{2}{*}{2} & Pasar Dargo & $\begin{array}{l}\text { Jl. Dargo- Jl. Dr. Cipto- Jl. } \\
\text { Brigjend Katamso- } \\
\text { Jl.Admodirono- Jl.Singosari } \\
\text { Raya- Jl. Sriwijaya- Jl.Veteran- } \\
\text { Jl.Kaligarang- Jl.Simongan- } \\
\text { Jl.Untung Suropati- TPA } \\
\text { Jatibarang }\end{array}$ \\
\hline & Pasar Karimata & $\begin{array}{l}\text { J1. Pancakarya- J1. RA. Kartini- } \\
\text { Dr. Cipto- Jl. Brigjend Katamso- } \\
\text { J1.Admodirono- Jl.Singosari } \\
\text { Raya- Jl. Sriwijaya- Jl.Veteran- } \\
\text { J1.Kaligarang- Jl.Simongan- } \\
\text { Jl.Untung Suropati- TPA } \\
\text { Jatibarang }\end{array}$ \\
\hline 3 & Rumah Pompa & $\begin{array}{l}\text { Jl. Barito- Jl. RA. Kartini- Dr. } \\
\text { Cipto- Jl. Brigjend Katamso- } \\
\text { Jl.Admodirono- Jl.Singosari } \\
\text { Raya- J1. Sriwijaya- Jl.Veteran- } \\
\text { Jl.Kaligarang- Jl.Simongan- } \\
\text { Jl.Untung Suropati- TPA } \\
\text { Jatibarang }\end{array}$ \\
\hline 4 & Mlatiharjo & $\begin{array}{l}\text { J1. Barito-Jl. Citarum-Jl. RA. } \\
\text { Kartini-Dr. Cipto- Jl. Brigjend } \\
\text { Katamso- Jl.Admodirono- } \\
\text { J1.Singosari Raya-Jl. Sriwijaya- } \\
\text { J1.Veteran-Jl.Kaligarang- } \\
\text { Jl.Simongan- Jl.Untung Suropati- } \\
\text { TPA Jatibarang }\end{array}$ \\
\hline 5 & Karang Tempel & $\begin{array}{l}\text { Jl. Barito-Jl. Halmahera Raya- Jl. Dr. } \\
\text { Cipto- Jl. Brigjend Katamso- } \\
\text { Jl.Admodirono- Jl.Singosari Raya- Jl. } \\
\text { Sriwijaya- Jl.Veteran-Jl.Kaligarang- }\end{array}$ \\
\hline
\end{tabular}




\begin{tabular}{|c|c|c|}
\hline & & $\begin{array}{l}\text { Jl.Simongan-Jl.Untung Suropati- TPA } \\
\text { Jatibarang }\end{array}$ \\
\hline & Mlatibaru & $\begin{array}{l}\text { Jl. Indragiri- Jl. Raden Patah- Jl. } \\
\text { Widoharjo- Jl. Dr. Cipto- Jl. Brigjend } \\
\text { Katamso-Jl.Admodirono- Jl.Singosari } \\
\text { Raya-Jl. Sriwijaya- Jl.Veteran- } \\
\text { Jl.Kaligarang- Jl.Simongan- Jl.Untung } \\
\text { Suropati- TPA Jatibarang } \\
\end{array}$ \\
\hline \multirow{3}{*}{6} & Tirtoyoso I & $\begin{array}{l}\text { Jl. Barito- Jl. RA. Kartini- Dr. Cipto- } \\
\text { Jl. Brigjend Katamso- Jl.Admodirono- } \\
\text { Jl.Singosari Raya- Jl. Sriwijaya- } \\
\text { Jl.Veteran- Jl.Kaligarang- } \\
\text { Jl.Simongan- Jl.Untung Suropati- TPA } \\
\text { Jatibarang }\end{array}$ \\
\hline & Tirtoyoso IV & \multirow{2}{*}{$\begin{array}{l}\text { Jl. Barito-Jl. Citarum- Jl. RA. Kartini- } \\
\text { Dr. Cipto- Jl. Brigjend Katamso- } \\
\text { Jl.Admodirono- Jl.Singosari Raya- Jl. } \\
\text { Sriwijaya- Jl.Veteran- Jl.Kaligarang- } \\
\text { Jl.Simongan- Jl.Untung Suropati- TPA } \\
\text { Jatibarang }\end{array}$} \\
\hline & Bugangan & \\
\hline
\end{tabular}

Looking at the results in Table 8, there is a changing of route of waste transportation which is choosing the shortest route by considering the flow of traffic. Shortest route selection is done by cutting the existing path by cutting Jalan Sriwijaya. The shortest route has a difference of one kilometer from the existing route. The distance difference is very good, because it avoids the point of congestion that is in the market area of Peterongan. The distance and time of transporting waste through the efficient route can be seen in Table 9 below :

Table 9. Distance and Waste Transportation Time After Efficiency in East Semarang Subdistrict

\begin{tabular}{|c|l|c|r|}
\hline $\begin{array}{c}\text { Truck } \\
\text { Number }\end{array}$ & $\begin{array}{c}\text { Temporary } \\
\text { Disposal Site }\end{array}$ & $\begin{array}{c}\text { Distance } \\
(\mathbf{k m})\end{array}$ & $\begin{array}{c}\text { Time } \\
\text { (minute) }\end{array}$ \\
\hline \multirow{3}{*}{1} & Pasar Rejomulyo & 33.4 & 58.8 \\
\cline { 2 - 4 } & Manisharjo & 31.8 & 63 \\
\cline { 2 - 4 } & Pasar Waru & 34 & 60.6 \\
\hline \multirow{2}{*}{2} & Pasar Dargo & 28.4 & 54.6 \\
\cline { 2 - 4 } & Pasar Karimata & 27.4 & 52.8 \\
\hline 3 & Rumah Pompa & 27 & 51.6 \\
\hline 4 & Mlatiharjo & 31.4 & 55.2 \\
\hline \multirow{2}{*}{5} & Karang Tempel & 25 & 49.8 \\
\cline { 2 - 4 } & Mlatibaru & 32.8 & 52.8 \\
\hline \multirow{2}{*}{6} & Tirtoyoso IV & 28.6 & 50.4 \\
\cline { 2 - 4 } & Tirtoyoso I & 28.8 & 55.8 \\
\cline { 2 - 4 } & Bugangan & 29 & 54.6 \\
\hline
\end{tabular}

The difference between the distance and time of the truck transport can be determined by comparing the distance and time of the existing route with the distance and time of the efficiency route. The difference between the distance and time of the existing route with the efficiency route can be seen in Table 10 below :

Table 10. Distance and Time Difference in East Semarang Subdistrict After Efficiency

\begin{tabular}{|c|c|c|c|}
\hline \multirow[b]{2}{*}{$\begin{array}{c}\text { Truck } \\
\text { Number }\end{array}$} & \multirow[b]{2}{*}{$\begin{array}{l}\text { Temporary } \\
\text { Disposal Site }\end{array}$} & \multicolumn{2}{|c|}{ Difference } \\
\hline & & $\begin{array}{c}\text { Distance } \\
(\mathbf{k m})\end{array}$ & $\begin{array}{c}\text { Time } \\
\text { (minute) }\end{array}$ \\
\hline \multirow{3}{*}{1} & Pasar Rejomulyo & 1 & 21 \\
\hline & Manisharjo & 1 & 18 \\
\hline & Pasar Waru & 1 & 18.6 \\
\hline \multirow{2}{*}{2} & Pasar Dargo & 1 & 26.4 \\
\hline & Pasar Karimata & 1 & 34.8 \\
\hline 3 & Rumah Pompa & 1 & 31.2 \\
\hline 4 & Mlatiharjo & 1 & 24.6 \\
\hline 5 & Karang Tempel & 1 & 34.8 \\
\hline
\end{tabular}

\begin{tabular}{|c|l|c|c|}
\hline \multirow{3}{*}{$\begin{array}{c}\text { Truck } \\
\text { Number }\end{array}$} & \multirow{2}{*}{$\begin{array}{c}\text { Temporary } \\
\text { Disposal Site }\end{array}$} & $\begin{array}{c}\text { Distance } \\
(\mathbf{k m})\end{array}$ & $\begin{array}{c}\text { Time } \\
(\text { minute) }\end{array}$ \\
\cline { 2 - 4 } & Mlatibaru & 1 & 26.4 \\
\hline \multirow{3}{*}{6} & Tirtoyoso I & 1 & 27,6 \\
\cline { 2 - 4 } & Tirtoyoso IV & 1 & 29,4 \\
\cline { 2 - 4 } & Bugangan & 1 & 31.8 \\
\hline
\end{tabular}

The result of distance and time difference calculation in Table 10 shows the result of the same distance difference, which is $1 \mathrm{~km}$ on all garbage trucks. While the largest time difference of 34.8 minutes is on trucks number 2 and number 5 that serve TPS Pasar Karimata and TPS Karang Tempel to TPA Jatibarang.

\section{Conclusion}

The existing condition of waste transportation system in Gayamsari and East Semarang Subdistricts can still be improved because the percentage of waste transportation service only reaches about $79 \%$. Effective and efficient waste transport system services are carried out with the change of shorter haulage routes and less traffic constraints, with the selection of the route with the consideration of traffic flow that is not crowded and wide access road. The shortest route and the fastest time selection is the first step to start a better waste transport system in these two districts.

This research was financially supported by The Faculty of Engineering, Diponegoro University, Indonesia through Strategic Research Grant 2018.

\section{References}

1. Tchobanoglous, G., Theisen, H., dan Vigil, S. (1993). Integrated Solid Waste Management. McGraw-Hill International Edition. New York.

2. Kao, J.J., dan Lin, T.I. (2002). Shortest Service Location Model for Planning Waste Pickup Locations. Journal of The Air \& Waste Management Association. 52:585-592.

3. Chu, Z., Xi, B., Song, Y., dan Crampton, E. (2013). Taking Out The Trash: Household Preferences Over Municipal Solid Waste Collection in Harbin, China. Habitat International. 40:194-200

4. Dinas Lingkungan Hidup (2016). Masterplan Persampahan 2016. Kota Semarang.

5. Yunita, A.T., dan Ali, M. (2014). Analisis Sistem Transportasi Sampah Kota Tuban Menggunakan Dynamic Programming. Jurnal Ilmiah Teknik Lingkungan Vol. 6 No. 1 :45-52.

6. Badan Pusat Statistik. (2017). Kecamatan Gayamsari Dalam Angka. Semarang.

7. Badan Pusat Statistik. (2017). Kecamatan Semarang Timur Dalam Angka. Semarang.

8. Damanhuri dan Tri Padmi. 2010. Diktat Kuliah Pengelolaan Sampah FTSL ITB. Bandung. 